\title{
Dentatorubral-Pallidoluysian Atrophy
}

National Cancer Institute

\section{Source}

National Cancer Institute. Dentatorubral-Pallidoluysian Atrophy. NCI Thesaurus. Code C122653.

A rare, autosomal dominant inherited progressive neurodegenerative disorder. It is caused by a mutation in the ATN1 gene, resulting in a combined degeneration of the dentatorubral and pallidoluysian systems. It can appear at any age, but it usually affects individuals between 20 and 30 years and leads to death within 10-15 years. The clinical presentation depends on the age of the affected individual; juvenile patients develop severe progressive myoclonus epilepsy and cognitive decline, whereas adult patients develop ataxia, choreoathetosis and dementia. 